\title{
Teste Triplo Biofisico: Um Novo Parâmetro Ultra-sonográfico Para Prognóstico em Gestações Iniciais
}

\author{
Biophysical Triple Test: A New Ultrasonographic Parameter
} for Prognostic Evaluation in Early Pregnancies

Joaquim R. M. Coelho, José Eduardo Chúfalo, Paula Contart

Ricardo Baruffi, José Gonçalves Franco Júnior

\section{RESUM0}

Objetivos: atrasos no desenvolvimento do saco gestacional (SG), no tamanho do botão embrionário (CCN), assim como freqüências cardiacas embrionárias (FCE) baixas podem ser considerados fatores de mau prognóstico na evolução da gestação. O objetivo deste trabalho foi avaliar a utilização destes 3 parâmetros em conjunto, o que denominamos Teste Triplo Biofisico (TTB).

Metodo: foram avaliadas 35 gestações únicas provenientes de fertilização assistida por injeção intracitoplasmática de espermatozóide (ICSI). Todos os exames de ultra-som foram realizados por um único examinador, após 4-5 semanas da transferência de embriões (6-7 semanas de gestação), com equipamento modelo Synergy da Diasonics, sonda transvaginal de 7,0 MHz. $O$ $S G$ foi medido em seu maior diâmetro transverso, o CCN foi medido no sentido sagital e a FCE pelo modo B-M e Doppler. Na análise estatística foi utilizado o teste de Fisher.

Resultados: considerou-se como parâmetros alterados : $S G<15,4 \mathrm{~mm}$; $C C N<3,9 \mathrm{~mm}$ e FCE $<100 \mathrm{bpm}$. Estes parâmetros foram calculados como média menos 1 desvio padrão das gestações em evolução $(n=32)$. As pacientes definidas com TTB positivo, isto é, as de risco para o aborto, foram aquelas que apresentaram pelo menos 2 parâmetros alterados. Das 35 gestações, o TTB foi positivo em 5, todas as 3 que abortaram e 2 que não abortaram. A diferença entre as que abortaram e as que evoluiram foi altamente significante $(p=0,0015$; $t$-Fisher). A sensibilidade do método foi de 100\%, com especificidade de 93,75\%, o que resultou em uma eficácia de 96,87\%.

Conclusão: o TTB é um método ultra-sonográfico não-invasivo e apresenta uma alta eficácia na avaliação prognóstica da gestação inicial.

PALAVRAS-CHAVE: Aborto. Ultra-sonografia. Fertilização in vitro.

\section{Introdução}

A ultra-sonografia transvaginal proporciona uma visão dinâmica do desenvolvimento embrionário e garante o delineamento detalhado

Centro de Medicina Fetal e Genética/Centro de Reprodução Humana da Maternidade Sinhá Junqueira das alterações anatômicas que ocorrem no primeiro trimestre de gravidez.

Habitualmente, a avaliação precoce das gestações subseqüentes à fertilização assistida ou de ciclo estimulado é feita entre 6 e 7 semanas, com o objetivo de se confirmar a presença de gravidez, o número de embriões em evolução, sua localização (tópica ou ectópica) e seu prognóstico.

Tem-se demonstrado que o saco gestacional (SG), o botão embrionário (CCN) e a frequência cardiaca embrionária ( $\mathrm{FCE}$ ) aumentam linearmente 
com a idade gestacional ${ }^{1,5,11}$.

Aproximadamente entre a $5^{\mathrm{a}}$ e $6^{\mathrm{a}}$ semanas, pode-se observar dentro do SG um complexo formado por vesícula vitelínica e embrião com batimento cardíaco. A medida do comprimento cabeça-nádega $(\mathrm{CCN})$ do embrião proporciona uma determinação precisa da idade gestacional ${ }^{6}$. A atividade cardíaca tem sido demonstrada a partir do $37^{\circ}$ dia do último período menstrual ${ }^{3}$.

Em geral, o embrião é visto com nitidez quando mede mais de $5 \mathrm{~mm}$, o que corresponde aproximadamente a 6 semanas e a FCE é superior a $90 \mathrm{bpm}^{10}$.

Atrasos no desenvolvimento destes parâmetros podem ser considerados como fatores de mau prognóstico na evolução da gestação. Freqüências cardiacas abaixo de $85 \mathrm{bpm}$ ou seu declínio observado em intervalo de poucos dias poderiam indicar um risco maior de abortamento espontâneo ${ }^{7,8,9}$.

O objetivo deste trabalho foi investigar, em conjunto, pela ultra-sonografia, os parâmetros biofísicos mais evidentes: medidas do SG, do CCN e da FCE e seu valor prognóstico em gestações iniciais provenientes de reprodução assistida, e que denominamos de Teste Triplo Biofísico (TTB).

\section{Pacientes e métodos}

Foram avaliadas prospectivamente, no período de fevereiro de 98 a julho de 98,35 gestações únicas, medindo-se o diâmetro do SG, o $\mathrm{CCN}$ e a FCE. Todas as gravidezes resultaram de fertilização assistida por injeção intracitoplasmática de espermatozóide (ICSI) .

Os exames de ultra-som foram realizados por um único examinador, 4-5 semanas após a transferência embrionária (6-7 semanas de gestação), com equipamento de ultra-som modelo Synergy da Diasonics, empregando-se sonda transvaginal de 7,0 $\mathrm{MHz}$, convexo de alta resolução. Foi usado modo B, B-M e Doppler para avaliação dos dados.

O SG foi medido em seu maior diâmetro transverso, o $\mathrm{CCN}$ foi medido no sentido sagital e a FCE pelo modo B-M e Doppler. $\mathrm{Na}$ análise estatística foi utilizado o teste de Fisher.

\section{Resultados}

As gestações em evolução ( $n=32)$ tiveram os parâmetros analisados pela média e desvio padrão (DP). A medida do saco gestacional (SG) apresentou média de 20,6 mm e DP de 5,2 $\mathrm{mm}$; a medida do botão embrionário (CCN) apresentou média de 4,9 $\mathrm{mm}$ e DP de $1,0 \mathrm{~mm}$ e a media da freqüência cardíaca embrionária (FCE) foi de 114,0 bpm e DP de 13,6 bpm.

As gestações que evoluíram para aborto $(n=3)$ apresentaram os resultados demonstrados na Tabela 1. Os parâmetros foram considerados alterados quando: $\mathrm{FCE}<100 \mathrm{bpm}, \mathrm{CCN}<3,9 \mathrm{~mm}$ e $\mathrm{SG}<15,4 \mathrm{~mm}$. Estes parâmetros foram obtidos subtraindo-se um desvio padrão da média das gestações que apresentaram evolução normal.

As pacientes com TTB positivo, isto é, aquelas com risco para aborto, foram as que apresentaram pelo menos 2 destes parâmetros alterados. Os resultados estão apresentados na Tabela 2, onde se observa que 5 pacientes tiveram o TTB positivo entre as quais se incluem todas as 3 que abortaram e 2 que não abortaram.

A diferença entre os dois grupos foi altamente significante, quando analisada estatisticamente pelo teste de Fisher ( $p=0,0015)$.

A sensibilidade do método foi de $100 \%$, com especificidade de $93,75 \%$, o que resulta em uma eficácia de $96,87 \%$. O valor preditivo positivo foi de $60 \%$ e o valor preditivo negativo de $100 \%$.

Tabela 1 - Resultados dos parâmetros analisados (medida do saco gestacional, tamanho do botão embrionário e freqüência cardíaca embrionária) nas pacientes que abortaram.

\begin{tabular}{lccr}
\hline Parâmetros analisados & Caso 1 & Caso 2 & Caso 3 \\
\hline Saco gestacional (mm) & 17,0 & 15,0 & 10,0 \\
Polo embrionário (mm) & 3,5 & 4,0 & 3,2 \\
Freqüência cardíaca & & & \\
embrionária (bpm) & 95 & 93 & 80 \\
\hline
\end{tabular}

Tabela 2 - Resultados dos parâmetros ultra-sonográficos analisados e número de pacientes que apresentaram aborto ou gravidez em evolução (não-aborto). Divisão em categorias: com pelo menos 2 parâmetros alterados e 1 ou nenhum parâmetro alterado (parâmetros analisados: medida do SG, CCN e FCE).

Parâmetros avaliados

Aborto
3
0
3

$\begin{array}{cc}\text { Não-aborto } & \text { Total } \\ 2 & 5 \\ 30 & 30 \\ 32 & 35\end{array}$

\section{Discussão}

Na gravidez normal, o batimento cardíaco é demonstrado pela ultra-sonografia transvaginal em embriões medindo em torno de 4-5 $\mathrm{mm}$ com saco gestacional maior que 6-9 $\mathrm{mm}$, que corresponderia 
a uma gestação de 5-6 semanas ${ }^{2}$.

Bromley et al. ${ }^{4}$ relataram que a discrepância na proporção das medidas entre o saco gestacional e embrião pode estar associada a morte fetal iminente. Coulam et al. ${ }^{5}$ observaram que gestações normais com saco gestacionais entre 20 e $30 \mathrm{~mm}$ devem conter embriões de pelo menos 2 a $5 \mathrm{~mm}$ com batimentos cardiacos entre 75 e $100 \mathrm{bpm}$.

A freqüência cardiaca embrionária tem sido o parâmetro individual mais valorizado para a análise do prognóstico das gestações iniciais. Alguns pesquisadores observaram que os embriões entre 6 e 9 semanas de gestação, com freqüência cardiaca menor que $85 \mathrm{bpm}$, têm tendência ao aborto espontâneo ${ }^{7}$. Em nossa casuística, as gestações que evoluíram para aborto apresentaram a frequência cardiaca embrionária entre 80 e 95 bpm nesta fase da gestação.

A maioria dos trabalhos a respeito da avaliação das gestações do primeiro trimestre, analisa os parâmetros morfológicos ultrasonográficos separadamente, como possiveis fatores prognósticos indicadores da ocorrência de aborto espontâneo. Entretanto, não se têm registros da análise conjunta destes parâmetros, um procedimento muito simples, mas que revelou resultados de excelente confiabilidade.

Conclui-se que o achado de pelo menos 2 parâmetros alterados entre a medida do saco gestacional, polo embrionário e freqüência cardiaca fetal indica uma alta chance de ocorrência de aborto, e quando somente 1 ou nenhum destes está alterado indica uma baixa chance de ocorrência de aborto.

O Teste Triplo Biofisico é um método ultrasonográfico não-invasivo altamente sensivel e específico como indicador do risco para abortamento em gestações iniciais, devendo pois seu uso ser considerado para integrar o exame de avaliação ultra-sonográfica tradicional.

\section{SUMMARY}

Purpose: it has been demonstrated that delays in gestational sac development, in crown-rump length and low embryonic heart rate could be indicators of poor pregnancy outcome. The purpose was to evaluate the use of these three parameters together, which we named Biophysical Triple Test (BTT).

Method: thirty-five singleton pregnancies following IVF treatment with intracytoplasmatic sperm injection (ICSI) were studied. All ultrasonographic scans were performed by the same examiner, 4-5 weeks after embryo transference (6-7 week gestation), using a Diasonics equipment, model Synergy, with a 7.0-MHz transvaginal transducer. The gestational sac (GS) was measured at the longest transverse diameter; the crownrump length (CRL) was measured at the sagittal plan and the embryonic heart rate (EHR) was calculated from frozen $B$ and M-mode and Doppler. The statistical test used was Fisher's test. Results: the following parameters were considered abnormal: $G S<15.4 \mathrm{~mm}, C R L<3.9 \mathrm{~mm}$, EHR $<100 \mathrm{bpm}$. The parameters were calculated from the means less one standard deviation for the ongoing pregnancies. The patients with a positive BTT, i.e., with a high risk of miscarriage, were those with at least 2 altered parameters. In 35 pregnancies, a positive BTT was found in 5 , all of those who miscarrided and 2 of those who had no miscarriage.

The difference between the two groups was highly significant ( $p=0.0015$; $t$-Fisher). The sensitivity of the method was is $100 \%$, with a specificity of $93.75 \%$, which resulted in a $96.87 \%$ efficacy.

Conclusion: the BTT is an ultrasonographic noninvasive method that presents high efficacy as an indicator of poor pregnancy outcome.

KEY WORDS: Miscarriage. Ultrasound. Gestational sac. Fertilization: in vitro.

\section{Referências}

1. Achiron R, Tadmor O, Mashiach S. Heart rate as a predictor of first-trimester spontaneous abortion after ultrasound-proven viability. Obstet Gynecol 1991; 78: 330-4

2. Bree RL, Edwards M, Bohm-Velez M, Beyler S, Roberts $J$, Mendelson EB. Transvaginal sonography in the evaluation of normal early pregnancy: Correlation with hCG level. AJR 1989; 153:75-9.

3. Britten S, Soenksen DM, Bustillo M, Coulam CB. Very early (24-56 days from last menstrual period) embryonic heart rate in normal pregnancies. Hum Reprod 1994; 9:2424-6.

4. Bromley B, Harlow BL, Laboda LA, Benacerraf BR. Small sac size in the first trimester: a predictor of poor fetal outcome. Radiology 1991; 178:375-7.

5. Coulam CB, Brittem S, Soenksem DM. Early (34-56 days from last menstrual period) ultrasonographic measurements in normal pregnancies. Hum Reprod 1996; 11:1771-4.

6. Degenhardt F, Bohmer S, Behrens O, Muhlhaus K. Transvaginale Ultraschallbiometrie der ScheitelSteiss-Länge im ersten Trimenon. Z Geburtshilfe Perinatol 1988;192:249-52.

7. Laboda LA, Estroff JA, Benacerraf BR. First trimester bradycardia. A sign of impending fetal loss. J Ultrasound Med 1989; 8:561-3.

8. May DA, Sturtevant NV. Embryonal heart rate as a predictor of pregnancy outcome: a prospective analysis. J Ultrasound Med 1991; 10:591-3.

9. Merchiers EH, Dhont M, De Sutter PA, Beghin CJ, Vandekerckhove DA. Predictive value of early embryonic cardiac activity for pregnancy outcome. Am J Obstet Gynecol 1991; 165:11-4.

10.Rempen A. Diagnosis of viability in early pregnancy with vaginal sonography. J Ultrasound Med 1990; 9:711-6.

11.Tezuka N, Sato S, Kanasugi H, Hiroi M. Embryonic heart rates: development in early first trimester and clinical evaluation. Gynecol Obstet Invest 1991; 32:210-2. 